\title{
CINÉTICA DE PIRÓLISE PARA O SORGO LIGNOCELULÓSICO: ANÁLISE DE DADOS TERMOGRAVIMÉTRICOS PARA APLICAÇÃO DO MODELO DE FRIEDMAN
}

\author{
Matheus H. B. P. da Silva ${ }^{a}$, Tiago J. P. de Oliveira ${ }^{\mathrm{b}}$, Carlos H. Ataíde ${ }^{\mathrm{c}}$, Daniel A. Cerqueira ${ }^{\mathrm{d}, \text {, }}$, Nádia G. Sousa ${ }^{\mathrm{a}}$ e Cássia \\ R. Cardoso \\ aDepartamento de Engenharia Química, Universidade Federal do Triângulo Mineiro, 38064-200 Uberaba - MG, Brasil \\ bepartamento de Engenharia, Universidade Federal de Lavras, 37200-000 Lavras - MG, Brasil \\ 'Faculdade de Engenharia Química, Universidade Federal de Uberlândia, 38408-100 Uberlândia - MG, Brasil \\ ${ }^{d}$ Departamento de Química, Universidade Federal do Triângulo Mineiro, 38064-200 Uberaba - MG, Brasil \\ 'Departamento de Engenharia de Alimentos, Universidade Federal do Triângulo Mineiro, 38064-200 Uberaba - MG, Brasil
}

Recebido em 23/04/2021; aceito em 26/07/2021; publicado na web em 27/08/2021

\begin{abstract}
PYROLYSIS KINETICS FOR LIGNOCELLULOSIC SORGHUM: THERMOGRAVIMETRIC DATA ANALYSIS FOR FRIEDMAN MODEL APPLICATION. Renewable resources are alternatives to fossil fuels and pyrolysis is identified as a process for the generation of biofuel products. The lignocellulosic sorghum is a cereal produced in brazil and it presents several applications, such as the production of second-generation ethanol. The present work aimed to study the slow pyrolysis of lignocellulosic sorghum, the biomass was characterized by means of proximate, ultimate, composition and carbohydrate analyzes. Thermogravimetric analyzes were performed with heating rates between 5 and $25 \mathrm{~K} \mathrm{~min}^{-1}$ and with $\mathrm{N}_{2}$ atmosphere. The Friedman model allows obtaining the kinetic parameters of biomass decomposition and is widely used due to its simplicity and precision; however, since it is sensitive to experimental noise, an algorithm was developed for the calculation of kinetic parameters for the slow pyrolysis of this biomass, enabling an accurate analysis of the experimental data. The activation energy found by the proposed methodology was $122.65 \pm 19.24 \mathrm{~kJ} \mathrm{~mol}^{-1}$, with determination coefficients above 0.97 . The comparison between experimental and theoretical data presented deviation values of $0.934 \%, 3.408 \%$ and $1.101 \%$ for heating rates of 5,10 and $15 \mathrm{~K} \mathrm{~min}^{-1}$, respectively, showing the accuracy for the determined kinetic parameters.
\end{abstract}

Keywords: activation energy; fibrous sorghum; pyrolysis; smoothing; Friedman model.

\section{INTRODUÇÃO}

Os avanços científicos e tecnológicos propiciam melhores índices de conforto e longevidade à população e, consequentemente, incentivam a procura por recursos energéticos, causando impactos ambientais que vêm sendo discutido mundialmente. Nesse sentido, a crescente preocupação com as questões ambientais e a conscientização mundial sobre a promoção do desenvolvimento em bases sustentáveis vêm estimulando a realização de pesquisas de desenvolvimento tecnológico que visam à identificação e à redução dos custos dessas tecnologias. ${ }^{1}$ As biomassas agrícolas são alternativas para a redução da dependência dos combustíveis fósseis. Dessa forma, biorrefinarias terão um importante papel na redução da poluição causada pelo uso de combustíveis fósseis e na garantia da sustentabilidade do setor químico. ${ }^{2}$

O sorgo possui várias aplicações, de acordo com suas variedades. Os tipos mais comuns de sorgos são o de forragem, grãos e o sorgo doce. A EMBRAPA Milho e Sorgo e algumas outras multinacionais, tais como Monsanto e Ceres, produzem variedades de sorgos fibrosos lignocelulósicos no Brasil, visando à futura produção de etanol de segunda geração. ${ }^{3} \mathrm{Na}$ produção de etanol de segunda geração, também chamado de etanol lignocelulósico, enzimas são usadas para gerar o combustível a partir da estrutura sólida da planta. Dependendo de fatores de mercado, o sorgo lignocelulósico também pode ser usado para a geração de energia quando utilizado em queimadores, ou na alimentação de processos de aquecimento na indústria alimentícia. ${ }^{3}$ Apesar do alto potencial produtivo do sorgo lignocelulósico, ainda são escassas as informações sobre

*e-mail: daniel.cerqueira@uftm.edu.br a recomendação de cultivares e o potencial de produtividade nas diversas regiões do Brasil. ${ }^{4}$

A pirólise é caracterizada pela degradação térmica do combustível sólido, que pode ser realizada na ausência total do agente oxidante ou em uma quantidade tal que a gaseificação não ocorra extensivamente. O processo de pirólise pode produzir óleo, carvão e gases, que podem atuar como combustíveis. ${ }^{5}$ As características principais do processo de pirólise lenta são tempos de residência de minutos ou horas e temperaturas entre 673 e $873 \mathrm{~K}$, gerando sólidos como o principal produto, um exemplo é o carvão ativado, utilizado com adsorvente e catalisador. A pirólise rápida ocorre com baixos tempos de residência $(0,5-5 \mathrm{~s})$ para os vapores pirolíticos, e moderadas temperaturas de reação, entre 673 e $873 \mathrm{~K}$, gerando como principal produto o bioóleo, que possui potencial aplicação como combustível em motores. ${ }^{6,7}$ A biomassa deve ser preparada antes do processo de pirólise, normalmente, a secagem e a moagem são os processos utilizados. ${ }^{8}$

O processo de pirólise é caracterizado com alto consumo energético e assim, apresenta altos custos operacionais. Para processos de pirólise rápida, aspectos como o sistema de controle do reator e as características de decomposição de biomassas com diferentes composições requerem investigações adicionais. ${ }^{9} \mathrm{O}$ preço final do bioóleo é alto e superior ao dos combustíveis fósseis, e o poder calorífico desse biocombustível é inferior ao dos combustíveis convencionais. A tecnologia atual encontra-se em busca da viabilidade econômica para a operação industrial. ${ }^{9}$ Já a pirólise lenta apresenta como possível produto o carvão ativado. O carvão ativado gerado a partir de biomassa apresenta menores custos de produção se comparado com o produto convencional e as carcterísticas do adsorvente são dependentes da composição inicial da biomassa e do seu processamento. ${ }^{10}$ Assim, estudos de caracterização de biomassas e otimização de processos 
devem ser realizados para que a pirólise de biomassa lignocelulósica seja melhor esclarecida e modificada em busca de redução de custos operacionais.

A análise termogravimétrica (TGA) é útil para avaliar processos de volatilização, resultando em medidas de alta precisão já que a temperatura e outras condições experimentais são bem controladas. As análises ocorrem com taxas de aquecimento de até $50 \mathrm{~K} \mathrm{~min}^{-1}$. Técnicas experimentais que envolvem altas taxas de aquecimento não asseguram resultados confiáveis devido às dificuldades em fornecer medições precisas de temperatura. ${ }^{11}$ Essa técnica permite avaliar o comportamento da biomassa durante a sua decomposição, determinar a estabilidade térmica do material, além de fornecer informações sobre as faixas de temperatura nas quais a decomposição é mais pronunciada. ${ }^{12} \mathrm{~A}$ análise permite ainda o cálculo dos parâmetros cinéticos para a decomposição do material.

A biomassa lignocelulósica é composta majoritariamente pelos biopolímeros celulose, hemicelulose e lignina, ${ }^{13,14}$ contendo menores quantidades de extrativos e sais inorgânicos. ${ }^{15}$ A lignina se decompõe em um amplo intervalo de temperatura, entre $500 \mathrm{~K} \mathrm{e}$ $1000 \mathrm{~K}$. A decomposição de hemiceluloses ocorre em um intervalo de temperatura de $400 \mathrm{~K}$ a $600 \mathrm{~K}$. A decomposição de celulose normalmente ocorre entre $500 \mathrm{~K}$ e $660 \mathrm{~K} \cdot{ }^{15,16}$ A Figura 1 mostra a importância das simulações e da obtenção dos parâmetros cinéticos para caracterização do processo de pirólise.

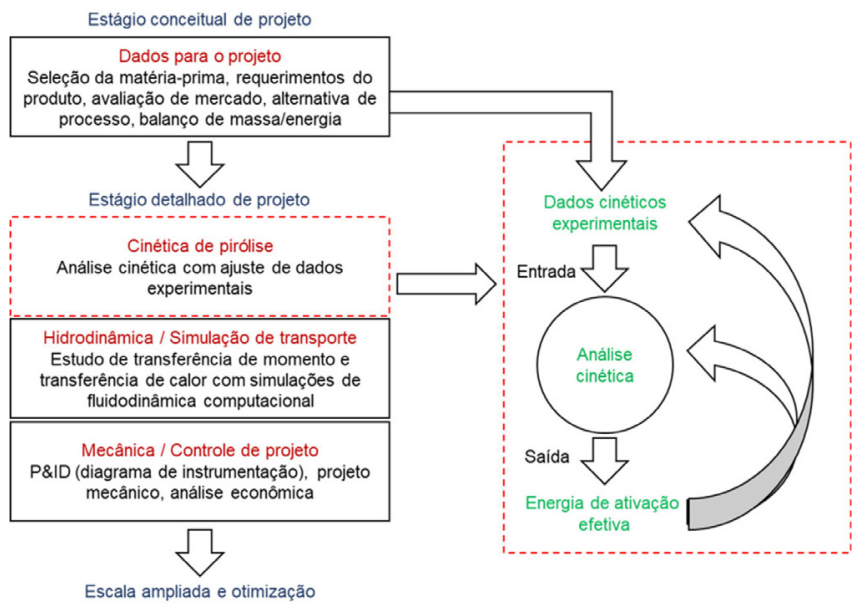

Figura 1. Fluxograma representando a importância da análise cinética para modelagem do processo pirolítico de biomassas, adaptado de Cai et al. ${ }^{19}$

A identificação de parâmetros cinéticos via termogravimetria depende das condições de pirólise, tais como temperatura, taxa de aquecimento, pressão, tamanho da partícula, gás de purga utilizado e presença de sais inorgânicos (cinzas) nos substratos. ${ }^{17}$ Fenômenos de transporte, como transferência de calor inter ou intra-partículas e de transferência de massa, também podem afetar o processo de volatilização. ${ }^{18}$ Para a determinação dos parâmetros cinéticos, há diversos modelos disponíveis que possibilitam o tratamento de dados, dentre eles o modelo de Kissinger, o modelo de KissingerAkahira-Sunose (KAS) e o modelo de Friedman, sendo possível a partir de todos a obtenção dos valores de energia de ativação $(E a)$. Dentre os métodos cinéticos isoconversionais, o método de Friedman é amplamente utilizado devido à sua simplicidade e precisão. ${ }^{19} \mathrm{Em}$ contrapartida, o uso desse modelo depende dos dados derivativos de conversão, o que pode favorecer a influência da sensibilidade aos ruídos. ${ }^{19} \mathrm{O}$ modelo de Friedman é descrito segundo a equação 1 .

$$
\ln \left(\beta \frac{d \alpha}{d T}\right)=\ln [A f(\alpha)]-\left(\frac{E a}{\mathrm{R}}\right)\left(\frac{1}{T}\right)
$$

em que $\beta$ é a taxa de aquecimento, $\alpha$ é a conversão, $T$ é a temperatura, $A$ é o fator pré exponencial de Arrhenius, $f(\alpha)$ é a função de conversão, $E a$ é a energia de ativação para a reação e R é a constante universal dos gases. A conversão $(\alpha)$ é dada pela equação 2 , sendo $m_{i}$ massa inicial, $m_{\alpha}$ massa em determinado tempo referente à conversão $\alpha$ e $m_{f}$ massa final.

$$
\alpha=\frac{m_{i}-m_{a}}{m_{i}-m_{f}}
$$

Considerando a determinação de parâmetros cinéticos, os dados experimentais podem se ajustar satisfatoriamente a diversos modelos, ao custo de calcular valores imprecisos..$^{20} \mathrm{O}$ modelo de Friedman é sensível aos ruídos de derivação, porém, o efeito dessa variação no cálculo isoconversional pode ser reduzido considerando não apenas valores isolados de conversão experimental, mas também considerando os dados de conversão vizinhos ao ponto experimental estudado, ${ }^{21}$ através da aplicação de métodos de suavização da curva de derivada. ${ }^{22}$ Assim, para garantir a confiabilidade dos resultados de parâmetros cinéticos deve-se adotar procedimentos de cálculo que considerem a sensibilidade aos ruídos, característica de modelos derivativos. Tais procedimentos consideram medição de linha de base experimental, seleção de dados experimentais e suavização da curva de derivada de conversão, como indicado pelo Comitê de Cinética da Confederação Internacional de Análises Térmicas e Calorimetria ${ }^{23} \mathrm{e}$ por trabalhos específicos sobre o modelo de Friedman. ${ }^{19}$

O objetivo desse trabalho é avaliar a pirólise lenta do sorgo lignocelulósico a partir dos dados experimentais de TGA, utilizando o modelo de Friedman, proposto em metodologia recentemente publicada em literatura por Cai et al. ${ }^{19}$ visando à determinação de parâmetros cinéticos precisos e considerando a redução da influência de ruídos experimentais no procedimento de cálculo. O trabalho é o primeiro a utilizar a metodologia proposta para determinação de parâmetros cinéticos para a pirólise lenta do sorgo lignocelulósico, uma biomassa ainda pouco explorada para tal propósito.

\section{PARTE EXPERIMENTAL}

\section{Amostragem e preparação do material}

O sorgo lignocelulósico híbrido, que foi desenvolvido na Monsanto foi utilizado nos testes termogravimétricos de pirólise. $\mathrm{O}$ colmo de sorgo foi primeiramente cortado em tamanhos de $20 \mathrm{~cm}$ e então moído em uma operação manual a fim de remover a grande parte de suco presente no material. O material foi então seco em um forno de circulação de ar por $24 \mathrm{~h}$ a $378 \mathrm{~K}$, depois foi processado em um moinho de facas para posterior classificação granulométrica. O material utilizado nas análises termogravimétricas foi a fração passante por peneira de 100 mesh. A utilização de partículas nessa granulometria busca minimizar a resistência à transferência de massa e de calor durante a análise. ${ }^{3}$ As análises de caracterização da biomassa, análise elementar, composição química, análise imediata e de carboidratos para o sorgo lignocelulósico foram realizadas em trabalho prévio, ${ }^{3}$ e os resultados são mostrados na Tabela 1 .

Os testes termogravimétricos do sorgo lignocelulósico foram realizados utilizando o analisador Shimadzu DTG-60H TGA/DTA. As medidas foram feitas usando purga de nitrogênio a $30 \mathrm{~mL} \mathrm{~min}^{-1}$. Amostras de aproximadamente $6 \mathrm{mg}$ foram analisadas nos testes.

O material foi primeiramente aquecido a $373 \mathrm{~K}$ com taxa de aquecimento constante de $50 \mathrm{~K} \mathrm{~min}^{-1}$ (a máxima taxa de operação do equipamento), essa temperatura foi mantida por $30 \mathrm{~min}$ a fim de eliminar a umidade. Depois da secagem, o material foi aquecido até $1173 \mathrm{~K}$ para avaliar sua degradação térmica. Diferentes taxas de 
Tabela 1. Análise elementar, composição química, análise imediata e de carboidratos para o sorgo lignocelulósico

\begin{tabular}{|c|c|c|c|c|c|c|c|}
\hline \multicolumn{2}{|c|}{ Análise elementar $\%(\mathrm{~m} / \mathrm{m})$} & \multicolumn{2}{|c|}{ Composição química $\%(\mathrm{~m} / \mathrm{m})$} & \multicolumn{2}{|c|}{ Análise imediata $\%(\mathrm{~m} / \mathrm{m})$} & \multicolumn{2}{|c|}{ Carboidratos $\%(\mathrm{~m} / \mathrm{m})$} \\
\hline $\mathrm{C}$ & 41,91 & Holocelulose* & 45,16 & Matéria volátil** & 77,84 & Arabinose & 2,56 \\
\hline $\mathrm{H}$ & 6,24 & Lignina & 11,30 & Cinzas** & 3,98 & Galactose & 1,06 \\
\hline $\mathrm{N}$ & 0,32 & Extrativos & 43,54 & Umidade** & 6,16 & Glicose & 68,61 \\
\hline $\mathrm{S}$ & 0,92 & & & Carbono fixo** & 18,18 & Xilose & 27,76 \\
\hline $\mathrm{O}$ & 50,61 & & & & & & \\
\hline
\end{tabular}

Nota: *Celulose e Hemiceluloses; **Base seca.

aquecimento foram utilizadas: 5, 10, 15, 20 e $25 \mathrm{~K} \mathrm{~min}^{-1}$. Diferentes taxas de aquecimento foram empregadas para minimizar os efeitos da transferência de massa e calor no cálculo dos parâmetros cinéticos. ${ }^{15}$ Adicionalmente, os testes foram feitos em triplicata. Dados de massa, tempo e temperatura foram registrados utilizando o software do equipamento, gerando dados de massa e temperatura a cada segundo de análise. Os dados correspondentes aos primeiros 30 min da reação não foram considerados; uma vez que nesse período há a perda de massa de água, antes do início da reação. ${ }^{24}$ Para cada análise foi considerada a linha de base referente à calibração da taxa de aquecimento usada, objetivando reduzir os efeitos de medição causados pela presença do gás de arraste. ${ }^{23}$

\section{Construção do algoritmo}

Para dados não-isotérmicos de TGA, é esperado uma tendência decrescente para os valores de massa, assim como uma tendência de acréscimo nos valores de temperatura. No entanto, há alguns pontos que não seguem essas tendências, devido aos erros sistemáticos do procedimento experimental; objetivando o maior detalhamento do comportamento de degradação da biomassa, utiliza-se o registro de dados a cada segundo de pirólise, o que pode gerar as variações de medida mencionadas. Tais erros podem causar a geração de uma curva de derivada de conversão com muitas flutuações. ${ }^{19}$

Visando reduzir a influência de tais flutuações, o tratamento de dados inclui a remoção dos pontos que não seguem as tendências de decréscimo para massa e de acréscimo para temperatura, além dos pontos do estágio de desidratação, com base em metodologia recentemente proposta. ${ }^{19}$

A derivada dos dados de conversão em função da temperatura pode ser calculada utilizando o método de diferenças finitas. A derivada da conversão pela temperatura foi calculada segundo a equação $3 .{ }^{19}$

$$
\left(\frac{d \alpha}{d T}\right)_{j}^{\exp }= \begin{cases}\frac{\alpha_{j+1}-\alpha_{j}}{T_{j+1}-T_{j}} & \text { para o ponto inicial } \\ \frac{1}{2} \frac{\alpha_{j}-\alpha_{j-1}}{T_{j}-T_{j-1}}+\frac{1}{2} \frac{\alpha_{j+1}-\alpha_{j}}{T_{j+1}-T_{j}} & \text { para os pontos intermediários } \\ \frac{\alpha_{j}-\alpha_{j-1}}{T T_{j-1}} & \text { para o ponto final }\end{cases}
$$

sendo $\alpha_{j}$ e $(d \alpha / d T)_{j}^{e x p}$, respectivamente, a conversão experimental e derivada de conversão experimental do ponto $j$-ésimo para o conjunto experimental de dados.

Considerando o modelo de Friedman, valores de $T, \alpha$ e $d \alpha / d T$ são utilizados para cálculo de $E a$. Usualmente, esses valores são calculados utilizando um método de interpolação. Alguns métodos de interpolação comumente utilizados são interpolação por pontos vizinhos, interpolação linear e o método de interpolação spline cúbico, o qual foi utilizado no presente trabalho, resultando em uma continuidade real entre os dados. Detalhes do método de interpolação spline cúbico são discutidos em literatura já mencionada. ${ }^{19} \mathrm{O}$ cálculo numérico foi feito utilizando o software Scilab ${ }^{\circledR}$ (versão 6.1.0).

O fluxograma para o algoritmo de remoção dos pontos que apresentavam erros é representado segundo a Figura 2. O algoritmo se inicia com a entrada dos dados experimentais de tempo, temperatura e massa; denominados de Old_t, Old_T e Old_m, respectivamente. Posteriormente, são criadas as variáveis que recebem os dados experimentais após a remoção dos pontos erráticos (New_t, New_T e New_m). Duas variáveis auxiliares (Aux_T e Aux_m) são necessárias para carregar o valor do ponto experimental que está sendo avaliado, que inicialmente, é o primeiro ponto do vetor de dados experimentais de temperatura e massa, respectivamente. Após essa inicialização, o algoritmo entra no laço de repetição, no qual a variável cont é inicializada com o valor zero e a variável i com o valor um. No interior desse laço excluem-se os valores de massa maiores ou iguais aos precedentes e os valores de temperatura menores ou iguais aos precedentes. $\mathrm{O}$ encerramento do processamento de dados ocorre quando a variável i é maior que o comprimento do vetor Old_t. Assim, após a finalização, o algoritmo fornece um novo conjunto de dados experimentais de tempo (New_t), temperatura (New_T) e massa (New_m).

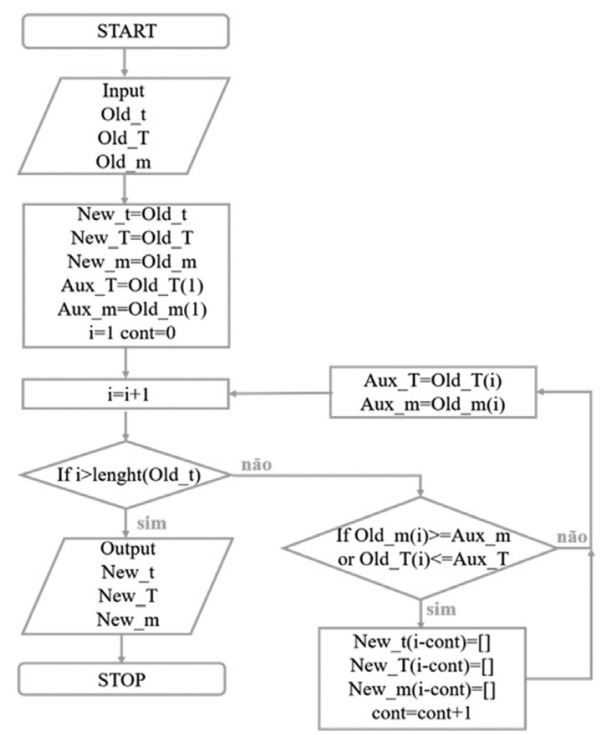

Figura 2. Fluxograma para a remoção de dados experimentais de massa crescente e temperatura decrescente, adaptado de Cai et al. ${ }^{19}$

Aliado ao método da remoção de dados erráticos, foi realizado o tratamento de dados segundo a metodologia proposta pelo Comitê de Cinética da Confederação Internacional de Análises Térmicas e Calorimetria, o qual indica a minimização dos efeitos de transferência de calor na escolha dos valores de taxas de aquecimento a serem utilizados para cálculo de parâmetros. Segundo o Comitê, os modelos isoconversionais para materiais lignocelulósicos são mais adequadamente aplicados para a etapa de 
volatilização dos componentes, ou seja, desconsiderando a etapa de carbonização que ocorre principalmente com a fração de lignina, a temperaturas mais elevadas. ${ }^{23}$ Assim, selecionou-se, para cada taxa de aquecimento utilizada, a faixa de temperatura referente à etapa de volatilização, ou seja, decomposição principal de hemicelulose e celulose.

A análise dos dados foi feita segundo o modelo de Friedman para as conversões de 0,05 a 0,95 com intervalo de 0,05 ; segundo orientação do Comitê..$^{23}$

\section{Suavização das curvas de derivadas}

O conjunto de dados derivativos apresentou ruídos, dessa forma foi feita a suavização da curva da derivada. Os métodos de ajuste não paramétricos comuns incluem a média móvel, SavitzkyGolay, e métodos de suavização de gráfico de dispersão localmente ponderada (LOWESS). ${ }^{19}$ No presente trabalho foi utilizado o método LOWESS, conforme sugerido pela literatura anterior. ${ }^{19}$ Para a suavização trabalha-se com os números de pontos de interrupção (n), o qual determina o número de intervalos e cada equação de regressão respectiva a estes intervalos, que, somadas, formam a curva completa.

Essa determinação de número de pontos de interrupção foi feita através do software Scilab ${ }^{\circledR}$ (versão 6.1.0), no qual há a disponível uma função na qual há a geração dos gráficos de suavização para um valor de $n$ fornecido. Dessa forma, foram feitos os testes para diferentes valores de $n$ e foi escolhido o valor que melhor representou o conjunto experimental de dados.

\section{Comparação entre os dados experimentais e teóricos}

Após a obtenção dos valores experimentais partindo-se da equação 1 , calcularam-se os valores de $d \alpha / d T$ para os valores teóricos pelo método de Runge-Kutta, por meio da equação 4.

$$
\left(\frac{d T}{d \alpha}\right)=\beta \mathrm{e}^{\frac{E a}{\mathrm{R} T}-\ln [A \alpha f(\alpha)]}
$$

sendo $\ln \left[A_{\alpha} f(\alpha)\right]$ o coeficiente linear da regressão da equação 1 para cada conversão.

Em posse dos dados experimentais (fornecidos pela equação 1) e dos dados teóricos (fornecidos pela equação 4), foi possível realizar a comparação entre os valores da derivada de conversão em função da temperatura através das equações 5 e 6.

$$
L S M=\sum_{j=1}^{z}\left|(y)_{j}^{\exp }-(y)_{j}^{\text {teórico }}\right|^{2}
$$

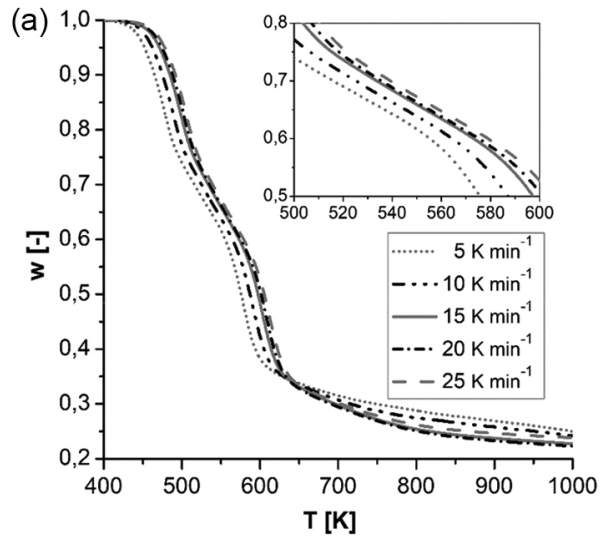

$$
A D P(\%)=100 \frac{\sqrt{\frac{L S M}{z}}}{y_{\max }}
$$

em que $z$ é o número de pontos utilizados, $j$ se refere aos dados referentes a cada ponto utilizado, $(y)_{j}^{\text {exp }}$ representa os valores experimentais de derivada de conversão em função da temperatura, $(y)_{j}^{\text {teórico }}$ indica os valores calculados de derivada de conversão em função da temperatura. $A D P$ representa o valor de desvio entre as curvas e $y_{\max }$ é o valor experimental máximo de derivada de conversão em função da temperatura.

Para o cálculo e geração de gráficos para o desvio entre os dados teóricos e experimentais, as conversões ( $\alpha$ ) utilizadas foram de 0,005 até 0,995 com intervalo de 0,005 ; pois a utilização de quantidade maior de dados de $E a, T$ e $\ln \left[A_{\alpha} f(\alpha)\right]$ permite que a curva teórica de $d \alpha / d T$ seja gerada de maneira detalhada, apresentando formatos e alturas de picos da curva mais precisos.

\section{RESULTADOS E DISCUSSÃO}

\section{Tratamento de dados}

O gráfico de fração mássica por temperatura é mostrado segundo a Figura 3 (a) e o gráfico da derivada de fração mássica por temperatura é apresentado pela Figura 3 (b).

A partir da Figura 3 (a) é possível notar que para as taxas de aquecimento 5, 10, $15 \mathrm{~K} \mathrm{~min}^{-1}$ não há sobreposição de curvas dentro do intervalo proposto, possibilitando uma análise não influenciada pelos efeitos de troca térmica. ${ }^{25}$ Por esse motivo, excluiu-se as taxas de aquecimento de 20 e $25 \mathrm{~K} \mathrm{~min}^{-1}$ para cálculo de parâmetros cinéticos.

As curvas de DTG da Figura 3 (b) mostram dois grandes picos, correspondendo à decomposição dos polímeros: hemicelulose, celulose e lignina. ${ }^{26}$ Considerando o intervalo de decomposição da hemicelulose ( 400 - $600 \mathrm{~K})$ e o intervalo de decomposição da celulose $(500-660 \mathrm{~K}),{ }^{15,16}$ e observando-se os picos gerados na Figura 3 (b), pode-se destacar que a etapa de volatilização engloba principalmente celulose e hemicelulose.

Para maiores taxas de aquecimento, há aumentos nas taxas de perda de massa. Essas mudanças podem ser atribuídas à maior diferença entre a temperatura do forno e da amostra, ${ }^{27}$ o que resulta em aceleração do processo de transferência de calor. ${ }^{28} \mathrm{~A}$ aceleração do processo de transferência de calor induz à sobreposição de picos para maiores taxas de aquecimento, devido à ocorrência de reações simultâneas que geralmente ocorrem separadamente em menores taxas de aquecimento. ${ }^{29} \mathrm{~A}$ escolha do intervalo de temperatura para cada taxa de aquecimento foi feita de modo a considerar o intervalo

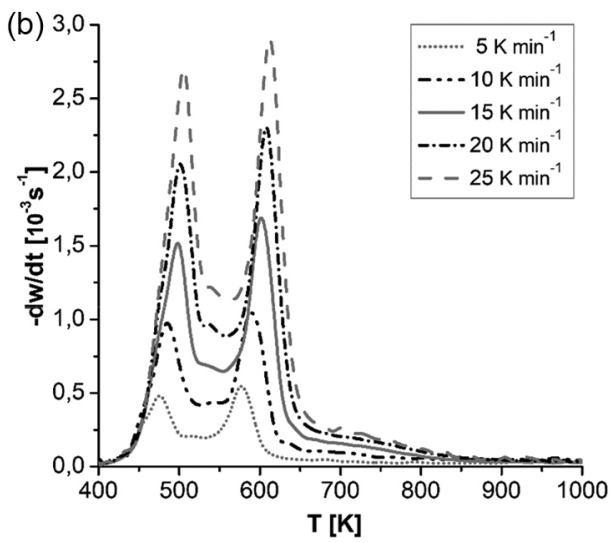

Figura 3. a) Fração mássica (w) versus temperatura; b) derivada da fração mássica por tempo (-dw/dt) versus temperatura 
que abrange toda a extensão dos picos para a derivada de fração mássica por temperatura, desconsiderando a etapa de carbonização.

Para a determinação dos parâmetros cinéticos considerou-se os intervalos de temperatura descritos com a intenção de eliminar o tempo de desidratação da biomassa e reduzir a influência de reações secundárias, as quais ocorrem a altas temperaturas e representam a degradação de produtos primários gerados em temperaturas mais baixas de reação pirolítica. ${ }^{23}$

A regressão linear foi feita segundo a equação 1, obtendo-se as retas de regressão, como mostra a Figura 4.

Os intervalos de temperatura utilizados para cada taxa de aquecimento são mostrados segundo a Tabela 2 .

Com a intenção de avaliar os resultados de energia de ativação e os resultados de coeficiente de determinação das regressões lineares para as três análises, os valores de energia de ativação versus conversão (Figura 5 (a)) e os valores de coeficientes de determinação versus conversão (Figura 5 (b)) são apresentados. Nos gráficos o termo "Análises 1" se refere ao primeiro conjunto de análises com taxas de aquecimento 5, 10 e $15 \mathrm{~K} / \mathrm{min}$; “Análises 2" representam as réplicas das análises e "Análises 3" as tréplicas.

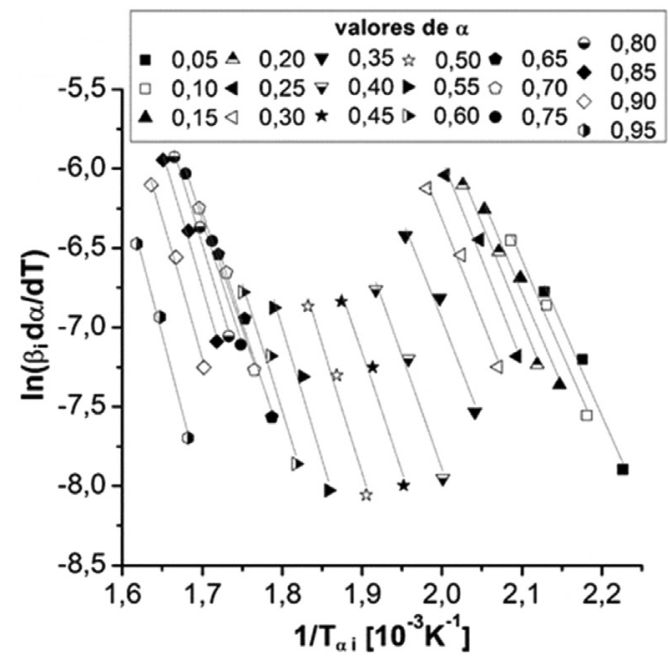

Figura 4. Inclinação para as conversões de 5 a 95\% do sorgo lignocelulósico

Os valores de $E a$ são variáveis para cada conversão e o valor médio encontrado foi de 122,65 $\pm 19,24 \mathrm{~kJ} \mathrm{~mol}^{-1}$.

Verifica-se na Figura 5 (a) que há similaridade de valores de $E a$ para as análises realizadas, uma vez que os conjuntos de dados apresentaram resultados próximos nas mesmas conversões para as três análises. As pequenas variações para cada conversão são esperadas,
Tabela 2. Seleção dos intervalos de temperatura para cada taxa de aquecimento da análise 1

\begin{tabular}{cc}
\hline Taxa de aquecimento $\left(\mathrm{K} \mathrm{min}^{-1}\right)$ & Intervalo de temperatura $(\mathrm{K})$ \\
\hline 5 & $430-610$ \\
10 & $440-620$ \\
15 & $450-630$ \\
\hline
\end{tabular}

tendo em vista que para materiais heterogêneos a diferença nos valores se deve à presença de reações com cinéticas diferentes para cada componente da biomassa. ${ }^{25}$

Há evidências de que o método utilizado retornou boas aproximações para o modelo de Friedman, uma vez que os valores $R^{2}$ se mantiverem em todas as regressões acima de 0,97 ; como mostra a Figura 5 (b).

Os valores de energia de ativação e de coeficiente de determinação podem ser comparados com trabalho prévio também utilizando o modelo de Friedman, feito para a cana-energia e que também considerou a etapa de volatilização para conversão de 0,05 a 0,95 . Esse estudo retornou valores de energia de ativação de 107,5 a 204 kJ mol $^{-1}$, com média geral de $177,1 \mathrm{~kJ} \mathrm{~mol}^{-1}$ e desvio padrão de $4,710 \%$, além de valores de coeficiente de determinação $\left(R^{2}\right)$ entre 0,9815 e 1,$0000 ;{ }^{30}$ que representam valores próximos aos encontrados para o sorgo lignocelulósico.

Cai et al. utilizaram o modelo de Friedman e exclusão dos pontos de acréscimo de massa no cálculo da energia de ativação para o caule do milho. Os autores encontraram valores entre 148 e $186 \mathrm{~kJ} \mathrm{~mol}^{-1}$ para o intervalo de conversão de 0,05 até 0,65 e valores de 186 a $473 \mathrm{~kJ} \mathrm{~mol}^{-1}$ utilizando intervalos de conversão de 0,65 até 0,85 . Os coeficientes de determinação variaram de 0,8898 até $1,0000 .{ }^{19}$

\section{Suavização das curvas de derivadas}

A curva experimental $d \alpha / d T$ sem ajustes é irregular e apresenta ruídos, dessa forma há a necessidade de suavizar essa curva para que a presença desses ruídos não interfira nos cálculos de energia de ativação.

Os gráficos de comparação da interpolação para diferentes valores de pontos de interrupção na taxa de aquecimento de $10 \mathrm{~K} \mathrm{~min}^{-1}$ da análise 1 são mostrados segundo a Figura 6.

Com a utilização de pontos de interrupção $(n)$ igual a 8 , a curva suavizada apresenta altura de picos diferentes da curva experimental. Para $n=20$, a curva suavizada representa de maneira eficiente os dados experimentais. Para $n=80$, a curva suavizada apresenta ruídos e não é adequada para a representação dos dados experimentais. Dessa maneira, após análise dos desvios entre curva experimental e curva
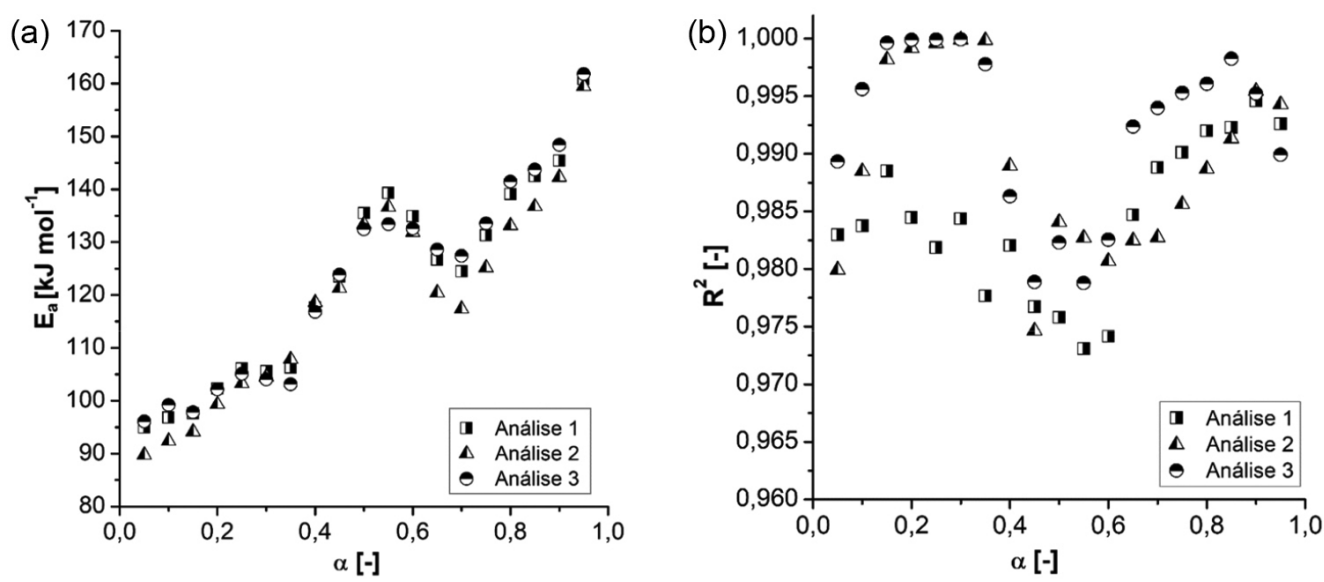

Figura 5. a) Energia de ativação por conversão para as três análises; b) coeficiente de determinação por conversão para as três análises 

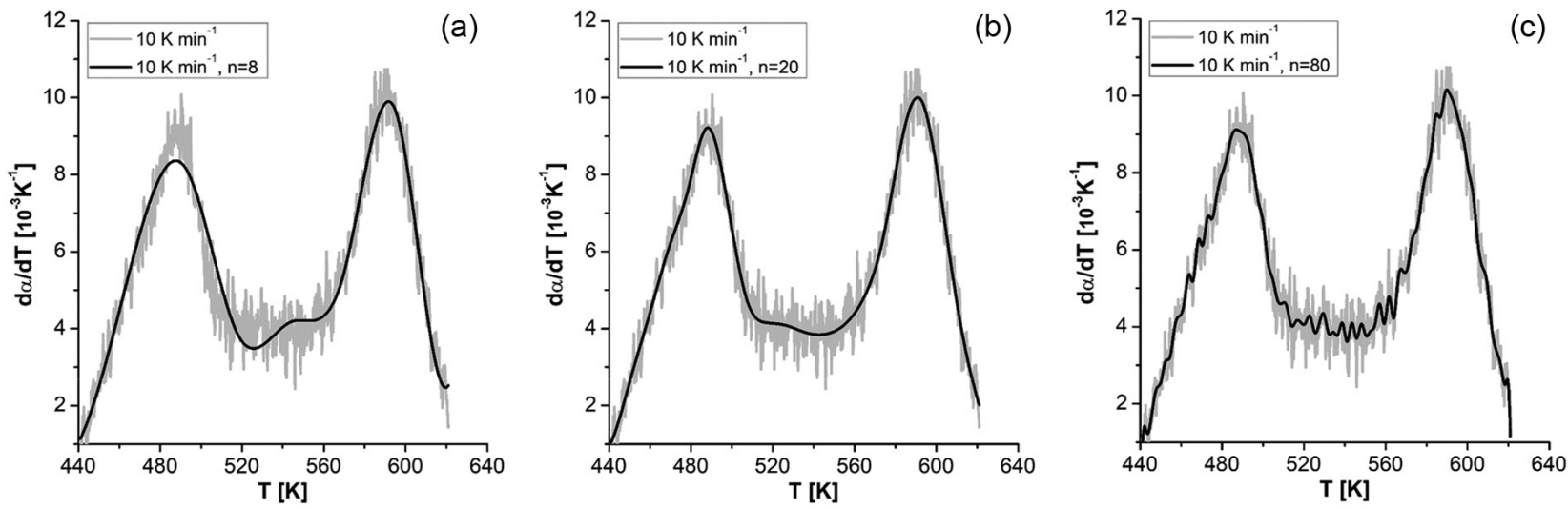

Figura 6. Suavização da curva de derivada de conversão em função da temperatura para a) 8 pontos de interrupção; b) 20 pontos de interrupção; c) 80 pontos de interrupção na taxa de aquecimento de $10 \mathrm{~K} \mathrm{~min}^{-1}$
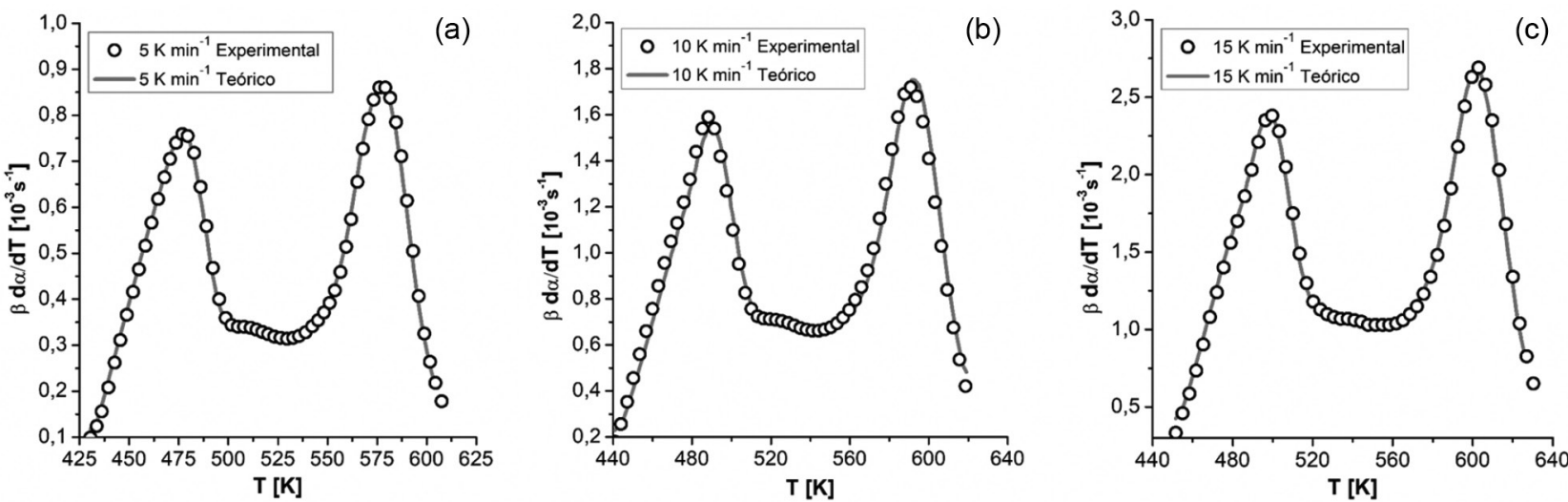

Figura 7. Curvas de dados experimentais e teóricos para a taxa de aquecimento de a) $5 \mathrm{~K} \mathrm{~min}^{-1}$; b) $10 \mathrm{~K} \mathrm{~min}^{-1}$ e c) $15 \mathrm{~K} \mathrm{~min}^{-1}$

suavizada foi utilizado o número de pontos de interrupção igual a 20, considerando a taxa de aquecimento de $10 \mathrm{~K} \mathrm{~min}^{-1}$.

\section{Comparação entre os dados experimentais e teóricos}

O cálculo para os dados teóricos de derivada de conversão em função da temperatura foi realizado pelo método de Runge-Kutta, segundo a equação 4. Os dados experimentais, ou seja, derivadas de conversão em função da temperatura após processo de suavização, foram comparados aos dados teóricos utilizando as equações 5 e 6 , obtendo-se os valores de $A D P$. Os valores de conversão utilizados para essa comparação foram do intervalo de 0,005 até 0,995 ; com passo de 0,005 (para obtenção de maior detalhamento); cada valor de conversão gerado nesse novo intervalo proposto gerou um valor de energia de ativação e um coeficiente linear para cada reta.

Os valores de $A D P$ entre as os dados experimentais e teóricos para as taxas de aquecimento 5,10 e $15 \mathrm{~K} \mathrm{~min}^{-1}$ obtidos são mostrados segundo a Tabela 3. Os gráficos de comparação entre os dados experimentais e teóricos para as taxas de aquecimento de 5, 10 e $15 \mathrm{~K} \mathrm{~min}^{-1}$ podem ser vistos na Figura 7.

Os ajustes obtidos apresentaram bons resultados, uma vez que os desvios padrão foram baixos. A remoção dos pontos inconsistentes

Tabela 3. Valores de desvio padrão entre os dados experimentais e teóricos da análise 1

\begin{tabular}{cc}
\hline Taxa de aquecimento $\left(\mathrm{K} \mathrm{min}^{-1}\right)$ & ADP $(\%)$ \\
\hline 5 & 0,934 \\
10 & 3,408 \\
15 & 1,101 \\
\hline
\end{tabular}

de massa e temperatura, incluindo o método de suavização, com a escolha adequada dos pontos de interrupção, mostrou-se relevante na obtenção desses resultados.

\section{CONCLUSÕES}

O modelo de Friedman foi utilizado para o estudo referente à pirólise lenta do sorgo lignocelulósico. O procedimento de remoção de pontos e suavização dos dados experimentais foi eficiente para calcular os valores de energia de ativação $(E a)$, possibilitando a obtenção de valores de $122,65 \pm 19,24 \mathrm{~kJ} \mathrm{~mol}^{-1}$; com os valores de coeficiente de determinação acima de 0,970 . Os parâmetros calculados resultam em uma representação precisa dos dados experimentais pelo modelo teórico, com os valores de desvio de $0,934 \% ; 3,408 \%$ e $1,101 \%$; para as taxas de aquecimento de 5,10 e $15 \mathrm{~K} \mathrm{~min}^{-1}$, respectivamente.

Os resultados são úteis para o manejo e aproveitando do sorgo lignocelulósico no processo de pirólise, indicando uma rota de processamento numérico dos dados termogravimétricos e fornecendo parâmetros para análises de simulação e otimização do processo.

\section{REFERÊNCIAS}

1. Freitas, G. S.; Dathein, R.; Revista Nexos Econômicos 2013, 7, 71.

2. Brehmer, B.; Boom, R.; Sanders, J.; Chem. Eng. Res. Des. 2009, 87, 1103.

3. Carvalho, W. S.; Oliveira, T. J.; Cardoso, C. R.; Ataíde, C. H.; Chem. Eng. Res. Des. 2014, 95, 337.

4. Fortes, C.; Evaristo, A. B.; Barros, A.; Pimentel, L. D.; Energia na Agricultura 2018, 33, 27. 
5. Klass, D. L.; Biomass for Renewable Energy, Fuels, and Chemicals; $1^{\text {st }}$ ed.; Academic Press: San Diego, 1998.

6. Goyal, H. B.; Seal, D.; Saxena, R. C.; Renewable Sustainable Energy Rev. 2008, 12, 504.

7. Mohan, D.; Pittman, C. U.; Philip, S.; Prog. Energy Combust. Sci. 2017, 62, 848 .

8. Tibola, F. L.; de Oliveira, T. J. P.; Carvalho, W. S.; Ataíde, C. H.; Cardoso, C. R.; Mater. Sci. Forum 2017, 899, 130.

9. Hu, X.; Gholizadeh, M.; J. Energy Chem. 2019, 39, 109.

10. Yahya, M. A.; Al-Qodah, Z.; Ngah, C. W. Z.; Renewable Sustainable Energy Rev. 2015, 46, 218.

11. Várhegyi, G.; Czégény, Z.; Jakab, E.; McAdam, K.; Liu, C.; J. Anal. Appl. Pyrolysis 2009, 86, 310 .

12. Santos, R. C. dos; Carneiro, A. C. O. de; Trugilho, P. F.; Mendes, L. M.; Carvalho, A. M. M. L.; Cerne 2012, 18, 143.

13. White, J. E.; Catallo, W. J.; Legendre, B. L.; J. Anal. Appl. Pyrolysis 2011, 91,1 .

14. Cai, J.; Wu, W.; Liu, R.; Renewable Sustainable Energy Rev. 2014, 36 , 236.

15. Cardoso, C. R.; Miranda, M. R.; Santos, K. G.; Ataíde, C. H.; J. Anal. Appl. Pyrolysis 2011, 92, 392.

16. Vamvuka, D.; Kakaras, E.; Kastanaki, E.; Grammelis, P.; Fuel 2003, 82, 1949.
17. Cardoso, C. R.; Meireles, C.; Ataíde, C. H.; ENEMP 2011 - XXXV Congresso Brasieiro de Sistemas Particulados, São Paulo, Brasil, 2011; pp. 653-660.

18. Fisher, T.; Hajaligol, M.; Waymack, B.; Kellogg, D.; J. Anal. Appl. Pyrolysis 2002, 62, 331

19. Cai, J.; Xu, D.; Dong, Z.; Yu, X.; Yang, Y.; Banks, S. W.; Bridgwater, A. V.; Renewable Sustainable Energy Rev. 2018, 82, 2705.

20. Sanchéz-Jiménez, P. E.; Pérez-Maqueda, L. A.; Perejón, A.; Criado, J. M.; Resour. Conserv. Recycl. 2013, 74, 75.

21. Huidobro, J. A.; Iglesias, I.; Alfonso, B. F.; Espina, A.; Trobajo, C.; Garcia, J. R.; Chemom. Intell. Lab. Syst. 2016, 151, 146.

22. Yu, Y.; Fu, X.; Yu, L.; Liu, R.; Cai, J.; J. Therm. Anal. Calorim. 2016, 124, 1641

23. Vyazovkin, S.; Burnham, A. K.; Criado, J. M.; Pérez-maqueda, L. A.; Popescu, C.; Sbirrazzuoli, N.; Thermochim. Acta 2011, 520, 1.

24. Cardoso, C. R.; Ataíde, C. H.; J. Anal. Appl. Pyrolysis 2013, 99, 49.

25. Lopes, F. C. R.; Tannous, K.; Thermochim. Acta 2020, 691, 178714.

26. Fermoso, J.; Mašek, O.; J. Anal. Appl. Pyrolysis 2018, 130, 358.

27. Uzun, B. B.; Yaman, E.; J. Energy Inst. 2017, 90, 825.

28. Li, H.; Li, L.; Zhang, R.; Tong, D.; Hu, C.; J. Energy Chem. 2014, 23, 732.

29. Tibola, F.; Oliveira, T. de; Cerqueira, D.; Ataíde, C.; Cardoso, C.; Quim. Nova 2020.

30. de Carvalho, V. S.; Tannous, K.; Thermochim. Acta 2017, 657, 56. 